\title{
Finite vertex primitive 2-arc regular graphs
}

\author{
X. G. Fang • C. H. Li · J. Wang
}

Received: 14 September 2005 / Accepted: 5 June 2006 /

Published online: 11 July 2006

(C) Springer Science + Business Media, LLC 2007

\begin{abstract}
A classification is given of finite graphs that are vertex primitive and 2arc regular. The classification involves various new constructions of interesting 2-arc transitive graphs.
\end{abstract}

Keywords 2-arc regular graphs · Vertex primitive

\section{Introduction}

Let $\Gamma$ be a finite undirected simple graph, and let $G \leq$ Aut $\Gamma$. The graph $\Gamma$ is called $G$-vertex primitive if $G$ acts primitively on the vertex set $V \Gamma$. An $s$-arc is a sequence $\left(\alpha_{0}, \ldots, \alpha_{s}\right)$ of $s+1$ vertices such that, for all $1 \leq i \leq s, \alpha_{i-1}$ is adjacent to $\alpha_{i}$, and for all $1 \leq i<s, \alpha_{i-1} \neq \alpha_{i+1}$. A graph $\Gamma$ is said to be $(G, s)$-arc transitive if $G \leq \mathrm{Aut} \Gamma$ is transitive on the set of $t$-arcs for each $t \leq s$. A $(G, s)$-arc transitive graph $\Gamma$ is called $(G, s)$-arc regular if $G$ acts regularly on the set of $s$-arcs of $\Gamma$, that is, no non-identity element of $G$ fixes an $s$-arc. The purpose of this paper is to classify graphs which are vertex primitive and 2-arc regular.

The study of $s$-arc transitive graphs was initiated by a celebrated result of Tutte [19] in 1949 when he proved that there exists no 6-arc transitive cubic graph. Since then, this class of graphs has received lots of attention. For example, Weiss in [21]

X. G. Fang $(\bowtie) \cdot J$. Wang

LMAM, School of Mathematical Sciences, Peking University, Beijing 100871, P. R. China

e-mail: xgfang@math.pku.edu.cn

J. Wang

e-mail: wangj@pku.edu.cn

C. H. Li

Department of Mathematics, Yunnan University, Kunming 650031, P. R. China

School of Mathematics and Statistics, The University of Western Australia,

Crawley, WA 6009, Australia

e-mail: li@maths.uwa.edu.au 
proved that there exists no 8-arc transitive graphs of valency at least 3; Praeger in [16] started a general analysis of automorphism groups of 2-arc transitive graphs. Refer to $[2,6,8,16]$ for more references.

Theorem 1.1. Let $\Gamma$ be a graph of valency $d$ such that a group $G \leq$ Aut $\Gamma$ is vertex primitive and 2-arc regular. Then one of the following holds:

(1) $\Gamma$ is a complete graph $K_{d+1}$, and $G$ is a sharply 3-transitive permutation group of degree $d+1$;

(2) $G=\mathbb{Z}_{2}^{n}: H$, where $H$ is a sharply 2-transitive group of odd degree;

(3) $G$ is almost simple, $G \triangleleft A:=$ Aut $\Gamma$, and exactly one row of Table 1 appears, where $\alpha$ is a vertex, and $n(A)$ is the number of non-isomorphic $G$-vertex primitive 2-arc transitive graphs with full automorphism group equal to A.

Remark on Theorem 1.1. Although for the four groups $G=F i_{24}, M,{ }^{2} D_{4}(3) .2$ or ${ }^{2} F_{4}(8) .3$, the precise number of corresponding graphs is not determined, the existence for all groups is known. This solves the existence problem for several primitive permutation groups that have a sharply 2-transitive subconstituent, which was unsettled in [20]. In particular, it excludes part (3) of the Main Theorem of [20] regarding the Baby Monster simple group and the Monster simple group.

Remark on Table 1. In the 9th row for $G=S_{p}, k$ is the number of prime divisors of $p-1$. The entries under the "ref." column refer to the lemmas where more detailed information about the graphs is given.

One of the main motivations for studying 2-arc regular graphs is that they are closely related to polygonal graphs, defined as follows: A graph $\Gamma$ is called a near-polygonal graph if there exists a number $m$ and a collection $\mathcal{C}$ of cycles of length $m$ in $\Gamma$ such that each 2 -arc of $\Gamma$ is contained in exactly one cycle in $\mathcal{C}$. If $m$ is the girth $g(\Gamma)$ of $\Gamma$ then the graph is called polygonal. Polygonal graphs are intriguing and hard to construct, refer to $[14,15]$ for references. By Corollary 1.2 of [10], a connected 2-arc regular graph $\Gamma$ is near-polygonal provided that for an arc $(\alpha, \beta)$ there exists an involution $g \in G$ such that $(\alpha, \beta)^{g}=(\beta, \alpha)$, leading to the constructions of several families of new polygonal graphs in [10]. A relation between 2-arc regular graphs and near-polygonal graphs was also found by Zhou [22]. We believe that with the 2-arc regular graphs constructed in this paper, more polygonal graphs can be produced.

This paper is organised as follows: Section 2 collects the notation and some preliminary results; in particular, it contains a reduction for proving Theorem 1.1 to the almost simple group case. In Section 3, all the candidates for the groups $G$ are given (in Table 2), and a series of technical lemmas are established. Finally, in Section 4, a proof of Theorem 1.1 is presented.

\section{Preliminaries}

The notation used in this paper is standard (see, for example, [1] and [4]). For two groups $K$ and $H, K . H$ is an arbitrary extension of $K$ by $H$, while $K: H$ stands for a split one. Moreover, the notation $K \circ H$ stands for the central product of the two groups. Sometimes, using the notation of the Atlas [1], by $p^{a}$ with $p$ prime we mean Springer 
Table 1 The almost simple case

\begin{tabular}{|c|c|c|c|c|c|c|}
\hline$G$ & $G_{\alpha}$ & $d$ & $A=\operatorname{Aut} \Gamma$ & $n(A)$ & Conditions & ref. \\
\hline \multirow[t]{2}{*}{$J_{1}$} & $7: 6$ & 7 & $J_{1}$ & 1 & & \\
\hline & $11: 10$ & 11 & $J_{1}$ & 1 & & \\
\hline$J_{3} .2$ & $19: 18$ & 19 & $J_{3} .2$ & 1 & & \\
\hline ON.2 & $31: 30$ & 31 & $O N .2$ & 3 & & 4.1 \\
\hline$J_{4}$ & $29: 28$ & 29 & $J_{4}$ & 1 & & \\
\hline$F i_{24}$ & $29: 28$ & 29 & $F i_{24}$ & 1 or 3 & & \\
\hline$M$ & $41: 40$ & 41 & $M$ & $\geq 1$ & & \\
\hline$A_{5}$ & $S_{3}$ & 3 & $S_{5}$ & 1 & & \\
\hline$A_{6} \cdot 2\left(\cong M_{10}\right)$ & $5: 4$ & 5 & $\operatorname{Aut}\left(A_{6}\right)$ & 1 & & \\
\hline $\begin{array}{l}S_{p}(p>5) \\
p \text { is prime }\end{array}$ & $Z_{p}: Z_{p-1}$ & $p$ & $S_{p}$ & $\begin{array}{l}2^{k-1}-1^{*} \\
2^{k}-1^{*} \\
2^{k+1}-1^{*}\end{array}$ & $\begin{array}{l}p \equiv 3(\bmod 4) \\
p \equiv 5(\bmod 8) \\
p \equiv 1(\bmod 8)\end{array}$ & 4.2 \\
\hline$S z(8)$ & $5: 4$ & 5 & $S z(8)$ & 1 & & \\
\hline$S z(8) .3$ & $7: 6$ & 7 & $S z(8) .3$ & 1 & & 4.1 \\
\hline${ }^{3} D_{4}(2) .3$ & $13: 12$ & 13 & ${ }^{3} D_{4}(2) .3$ & 1 or 3 & & \\
\hline${ }^{2} F_{4}(2)$ & $13: 12$ & 13 & ${ }^{2} F_{4}(2)$ & 1 & & \\
\hline${ }^{2} F_{4}(8) .3$ & $37: 36$ & 37 & ${ }^{2} F_{4}(8) .3$ & $\geq 1$ & & 4.1 \\
\hline$L_{3}(4)$ & $3^{2}: Q_{8}$ & 9 & $L_{3}(4) \cdot D_{12}$ & 1 & & \\
\hline$S_{4}(4) .4$ & $17: 16$ & 17 & $S_{4}(4) .4$ & 1 & & 4.3 \\
\hline$U_{3}(4) .4$ & $13: 12$ & 13 & $U_{3}(4) .4$ & 1 & & \\
\hline$L_{2}(q)$ & & & $P G L_{2}(q)$ & 1 & $\varepsilon= \pm 1$ & \\
\hline$q$ is prime, & $A_{4}$ & 4 & $L_{2}(q)$ & $(q-\varepsilon) / 12$ & $q \equiv \varepsilon(\bmod 4)$ & [9] \\
\hline $\begin{array}{c}q \equiv \pm 3(\bmod 8) \\
\not \equiv \pm 1(\bmod 10)\end{array}$ & & & $\begin{array}{l}P G L_{2}(q) \\
L_{2}(q)\end{array}$ & $\begin{array}{l}1 \\
(q-\varepsilon-2) / 6\end{array}$ & $\begin{array}{l}\varepsilon= \pm 1 \\
q \not \equiv \varepsilon(\bmod 4)\end{array}$ & \\
\hline $\begin{array}{l}U_{3}\left(2^{r}\right) \\
r>3 \text { is prime }\end{array}$ & $3^{2}: Q_{8}$ & 9 & $U_{3}\left(2^{r}\right) . S_{3}$ & $\left(2^{r-1}-1\right) / r$ & & 4.4 \\
\hline$L_{3}(q)$ & & & $L_{3}(q)$ & 1 & $q \equiv 3(\bmod 4)$ & \\
\hline $\begin{array}{l}q \text { is prime, } \\
q \equiv 4,7(\bmod 9)\end{array}$ & $3^{2}: Q_{8}$ & 9 & $\begin{array}{l}L_{3}(q) \\
L_{3}(q) \cdot S_{3}\end{array}$ & $\begin{array}{l}2 \\
1\end{array}$ & $q \equiv 1(\bmod 4)$ & 4.5 \\
\hline$U_{3}(q)$ & & & $U_{3}(q) .2$ & 1 & $q \equiv 3(\bmod 4)$ & \\
\hline $\begin{array}{l}q \text { is prime, } \\
q \equiv 2,5(\bmod 9)\end{array}$ & $3^{2}: Q_{8}$ & 9 & $\begin{array}{l}U_{3}(q) .2 \\
U_{3}(q) .6\end{array}$ & $\begin{array}{l}2 \\
1\end{array}$ & $q \equiv 1(\bmod 4)$ & 4.6 \\
\hline
\end{tabular}


an elementary abelian group of order $p^{a}$, by $[n]$ for an integer $n$ we mean an arbitrary group of order $n$, and simply by $n$ denote a cyclic group of order $n$.

Let $\Gamma$ be a graph of valency $d$, and let $G \leq$ Aut $\Gamma$ be vertex primitive and 2-arc regular on $\Gamma$. Then for a vertex $\alpha, G_{\alpha}$ acts sharply 2-transitively on $\Gamma(\alpha)$. Hence $\Gamma$ is an orbital graph of $G$ acting on $V \Gamma$, and $\Gamma$ corresponds to a sharply 2-transitive subconstituent. By a result of the third author [20], we have the following lemma:

Lemma 2.1. Using the notation defined above, one of the following holds:

(i) $G$ acts 2-transitively on $V \Gamma$, and $\Gamma$ is a complete graph,

(ii) $G$ is affine, that is, $Z_{p}^{n} \triangleleft G \triangleleft \operatorname{AGL}(n, p)$, where $p$ is a prime;

(iii) $G$ is an almost simple group.

Next we treat parts (i) and (ii).

Lemma 2.2. If $\Gamma$ is a complete graph or $G$ is of affine type, then Theorem 1.1 holds.

Proof: Suppose first that $\Gamma$ is a complete graph. Since $G$ is transitive on the set of 2-arcs of $\Gamma$, it follows that $G$ is transitive on the set of all triples of vertices of $V \Gamma$. Hence $G$ is 3-transitive on $V \Gamma$. Further, since $G$ is regular on the set of 2-arcs of $\Gamma$, it follows that $G$ is regular on the set of triples of vertices of $V \Gamma$, so $G$ is sharply 3-transitive on $V \Gamma$, as in part (1) of Theorem 1.1.

Assume next that $G$ is affine, that is, $Z_{p}^{n} \triangleleft G \triangleleft \operatorname{AGL}(n, p)$, where $p$ is a prime. Then $G=N: H$, where $N=Z_{p}^{n}$, and $H=G_{\alpha}$ is irreducible on $N$. Since $G$ is regular on the set of 2-arcs, $G_{\alpha}$ is a sharply 2-transitive permutation group on $\Gamma(\alpha)$. Because $N$ is regular on $V \Gamma$, vertices of $\Gamma$ may be identified with elements of $N$. Further, let $\alpha$ be the vertex corresponding to the identity of $N$. Then the subset $S:=\Gamma(\alpha)$ is such that two vertices $x, y$ are adjacent if and only if $y x^{-1} \in S$. In this identification, $H$ acts on $V \Gamma=N$ by conjugation. Since $H$ is 2-transitive on $S$, it follows that $p=2$ and $N$ is an elementary abelian 2-group, as in part (2) of Theorem 1.1.

This reduces the proof of Theorem 1.1 to the case where $G$ is an almost simple group. To investigate this case, we need more notation.

For a group $G$ and a core free subgroup $H<G, G$ has a faithful transitive permutation representation on the set of right cosets $[G: H]:=\{H x \mid x \in G\}$ by right multiplication. A coset graph

$$
\Gamma=\operatorname{Cos}(G, H, H g H), \text { where } g \in G \text { with } g^{2} \in H
$$

is defined as the graph with vertex set $[G: H]$ such that $H x$ is adjacent to $H y$ if and only if $y x^{-1} \in H g H$, denoted by $\operatorname{Cos}(G, H, H g H)$.

It follows from the definition that if $\langle H, g\rangle=G$ then $\Gamma$ is connected. Label the vertices corresponding to $H$ and $H g$ to be $\alpha, \beta$, respectively. Then the vertex stabilisers $G_{\alpha}=H$ and $G_{\beta}=H^{g}$, and the arc stabiliser $G_{\alpha \beta}=G_{\alpha} \cap G_{\beta}=H \cap H^{g}$. It is easily shown that $\Gamma$ is $(G, 2)$-arc transitive if and only if $G_{\alpha}$ is 2-transitive on the neighborhood $\Gamma(\alpha)$. The following simple lemma then follows, refer to [2].

Lemma 2.3. A coset graph $\Gamma=\operatorname{Cos}(G, H, H g H)$ is $(G, 2)$-arc transitive if and only if $H$ acts 2-transitively on the set of cosets $\left[H: H \cap H^{g}\right]$. 
Table 2 The candidates for $G$

\begin{tabular}{|c|c|c|c|}
\hline$G$ & $H=K: L$ & $d$ & Remark \\
\hline \multirow[t]{2}{*}{$J_{1}$} & $11: 10$ & 11 & \\
\hline & $7: 6$ & 7 & \\
\hline$J_{3} .2$ & $19: 18$ & 19 & \\
\hline$O N .2$ & $31: 30$ & 31 & \\
\hline$J_{4}$ & $29: 28$ & 29 & \\
\hline$F i_{24}$ & $29: 28$ & 29 & \\
\hline$M$ & $41: 40$ & 41 & \\
\hline$M_{10}$ & $5: 4$ & 5 & \\
\hline$S_{p}$ & $Z_{p}: Z_{p-1}$ & $p$ & $p$ is prime \\
\hline$S z(8)$ & $5: 4$ & 5 & \\
\hline$S z(8) .3$ & $7: 6$ & 7 & \\
\hline${ }^{3} D_{4}(2) \cdot 3$ & $13: 12$ & 13 & \\
\hline${ }^{2} F_{4}(2)$ & $13: 12$ & 13 & \\
\hline${ }^{2} F_{4}(8) .3$ & $37: 36$ & 37 & \\
\hline${ }^{2} G_{2}(27) \cdot 3$ & $19: 18$ & 19 & \\
\hline$L_{3}(4)$ & $3^{2}: Q_{8}$ & $3^{2}$ & \\
\hline$S_{4}(4) .4$ & $17: 16$ & 17 & \\
\hline$U_{3}(4) .4$ & $13: 12$ & 13 & \\
\hline$U_{3}\left(2^{r}\right)$ & $3^{2}: Q_{8}$ & 9 & $r>3$ is prime \\
\hline$L_{3}(q)$ & $3^{2}: Q_{8}$ & 9 & $q \equiv 4,7(\bmod 9)$ is prime \\
\hline$U_{3}(q)$ & $3^{2}: Q_{8}$ & 9 & $(5<) q \equiv 2,5(\bmod 9)$ is prime \\
\hline
\end{tabular}

\section{Some technical lemmas}

Let $G$ be an almost simple group that acts on a graph $\Gamma$ vertex primitively and 2-arc regularly. We give a series of lemmas regarding the group $G$ and its action. The first lemma lists the candidates of $G$ and $G_{\alpha}$, where $\alpha$ is a vertex.

Lemma 3.1. Let $\Gamma$ be a non-complete graph of valency d. Assume that $G \leq$ Aut $\Gamma$ acts on $V \Gamma$ primitively and on the set of 2-arcs regularly. Assume further that $G$ is almost simple. Then one of the following holds:

(i) $d=3, G=A_{5}$, and $\Gamma$ is the Peterson graph;

(ii) $d=4, G=P S L(2, p)$ with $p$ prime such that $p \equiv \pm 3(\bmod 8)$, and $5 \neq p \neq \equiv$ $\pm 1(\bmod 10)$; in this case there are exactly $[(p+\varepsilon) / 12]$ non-isomorphic graphs, where $\varepsilon= \pm 1$ such that 3 divides $p \pm \varepsilon$;

(iii) $d \geq 5$, and $(G, H, d)$ is one of the entries listed in Table 2; furthermore, for each $G$ in the list, the choice of $H$ (up to $G$-conjugacy) is unique, where $H=G_{\alpha}$ is the vertex stabiliser.

Proof: If $d \leq 4$, this case is classified in [9] from which we have parts (i) and (ii). 
Assume next $d \geq 5$. Since $G$ is regular on the 2 -arcs of $\Gamma$, the stabiliser $H$ is faithful and sharply 2-transitive on $\Gamma(\alpha)$. By the result of [20], we have one of the following:

(i) $G=M$ or $B M$, and $H$ is a maximal 2-local subgroup of $G$,

(ii) $G=U_{3}(8) .6$ or $S_{4}(8) . S_{3}$, and $H=19: 18$ or $\left(D_{14} \times D_{14}\right) .2 . S_{3}$, respectively;

(iii) or $G$ is a group in Table 2 .

In case (i), we must have $H=Z_{2}^{t}: L$ for some integer $t \geq 3$. We claim that $Z_{2}^{t}$ is a Sylow 2-subgroup of $G$. If this is not the case then there is a Sylow 2-subgroup $P$ such that $Z_{2}^{t}<P$. Clearly, $Z_{2}^{t}<\mathbf{N}_{P}\left(Z_{2}^{t}\right)$. Set $T:=\left\langle\mathbf{N}_{P}\left(Z_{2}^{t}\right), H\right\rangle$. Then $H$ is a proper normal subgroup of $T$. Note that $G$ is simple. Thus $T<G$, which contradicts the fact that $H$ is a maximal subgroup of $G$. On the other hand, by [1] we know that a Sylow 2-subgroup of $G$ is not elementary abelian, which implies that $G$ is neither $M$ nor $B M$.

If $G=U_{3}(8) .6$, then $H=K: L=19: 18$. Computation shows that $\left|\mathbf{N}_{G}(L)\right|=54$. It follows that there is no 2-element $g$ satisfying Lemma 2.3, and hence no graph occurs in this case.

If $G=S_{4}(8) \cdot S_{3}$, then $H=K: L=\left(D_{14} \times D_{14}\right) \cdot 2 \cdot S_{3}$ and $K=7^{2}$. It is clear that there exists an involution in $L$ which commutes with one of the two direct factors of $K$. However, this contradicts the fact that $L$ is regular on $K$. Hence no graph occurs in this case. So $(G, H, d)$ is one of the triples given in Table 2 .

The next lemma regards all 2-elements $g$ that, together with $(G, H)$ given in Table 2, give rise to required graphs.

Lemma 3.2. Let $(G, H)$ be one of the pairs in Table 2. Assume that the set

$$
I(G, L)=\left\{x \in \mathbf{N}_{G}(L) \backslash L \mid x \text { is a 2-element, and } x^{2} \in L\right\}
$$

is not empty. Then $\operatorname{Cos}(G, H, H g H)$ is $G$-vertex primitive and 2-arc regular if and only if $g \in I(G, L)$.

Proof: Let $g \in I(G, L)$, and $\Gamma=\operatorname{Cos}(G, H, H g H)$. Since $\mathbf{N}_{H}(L)=L$ and $g \notin L$, we have $g \notin H$. Thus $\langle H, g\rangle=G$ since $H$ is a maximal subgroup of $G$, and further, since $H$ is sharply 2 -transitive on $[H: L], \Gamma$ is $(G, 2)$-arc regular. Conversely, if $\Gamma=\operatorname{Cos}(G, H, H g H)$ is $G$-vertex primitive and 2-arc regular, then it follows from Lemma 2.3 that $g \in I(G, L)$.

The next lemma is crucial for determining the full automorphism group Aut $\Gamma$.

\begin{tabular}{llllll}
\hline$G$ & $G_{\alpha}=H$ & $F$ & $F_{\alpha}$ & $|V \Gamma|$ & $|\Gamma(\alpha)|$ \\
\hline$A_{6} \cdot 2$ & $5: 4$ & $S_{6}$ wr 2 & $S_{5}$ wr 2 & 36 & 5 \\
$S_{7}$ & $7: 6$ & $A_{9}$ & $L_{2}(8): 3$ & 120 & 7 \\
$S_{p}$ & $Z_{p}: Z_{p-1}$ & $S_{p+1}$ & $L_{2}(p): 2$ & $(p-2) !$ & $p$ \\
$L_{3}(4)$ & $3^{2}: Q_{8}$ & $U_{4}(3)$ & $3_{+}^{1+4}: 2 S_{4}$ & 280 & 9 \\
$S z(8) .3$ & $7: 6$ & $A_{65}$ & $S_{63}$ & 2080 & 7 \\
$S z(8) .3$ & $7: 6$ & $S p_{4}(8)$ & $O_{4}^{+}(8)$ & 2080 & 7 \\
& & & & & \\
\hline
\end{tabular}


Lemma 3.3. Let $(G, H, L)$ be a triple given in Table 2 , and let $\Gamma=\operatorname{Cos}(G, H, H g H)$ for some $g \in I(G, L)$, where $I(G, L)$ is as defined in Lemma 3.2. Then $G$ is normal in Aut $\Gamma$, and $\operatorname{Aut} \Gamma \leq \operatorname{Aut}(G)$. Moreover, if $G_{1} \leq \operatorname{Aut} \Gamma$ and $G_{1} \cong G$, then $G_{1}=G$.

Proof: Let $A=A u t \Gamma$, and let $T=\operatorname{soc}(G)$, the socle of $G$. Then $T \unlhd G \leq A \leq$ $\operatorname{Sym}(V \Gamma)$. Suppose that $G$ and $A$ have different socles. It follows that there exist groups $F_{1}$ and $F$ such that $G \leq F_{1}<F \leq \operatorname{Sym}(V \Gamma), \operatorname{soc}(G)=\operatorname{soc}\left(F_{1}\right) \neq \operatorname{soc}(F)$, and $F_{1}$ maximal in $F$. Noticing that $G$ is almost simple and primitive on $V \Gamma$, all the possibilities for $F_{1}, F$ are listed in [11, Tables 2-6]. It then follows that one of the entries in the following table holds, where $\alpha$ is a vertex:

Notice that, since $G \leq F \leq \operatorname{Aut} \Gamma$, the overgroup $F$ has a 2-transitive subconstituent $F_{\alpha}^{\Gamma(\alpha)}$ of degree $|\Gamma(\alpha)|$. It is then easily shown that only in row $4, F_{\alpha}$ could have a transitive permutation representation of degree $|\Gamma(\alpha)|$. In this case, $F_{\alpha}=3_{+}^{1+4}: 2 S_{4}$ and $F_{\alpha}^{[1]}=\left[3^{3}\right]$. Now $\left(F_{\alpha}^{[1]}\right)^{\Gamma(\beta)} \triangleleft F_{\alpha \beta}^{\Gamma(\beta)} \cong 2 S_{4}$, which is not possible. Thus in all the cases, we get a contradiction, $\operatorname{sos} \operatorname{soc}(A)=\operatorname{soc}(G)$. Checking the groups listed in Table 2 , it is easily shown that $G \unlhd \operatorname{Aut}(T)$, so $\operatorname{Aut}(T)=\operatorname{Aut}(G)$.

The following lemma deals with the isomorphism problem for our graphs.

Lemma 3.4. Suppose that $(G, H, L)$ is a triple given in Table 2. Then, for any $g, g^{\prime} \in$ $I(G, L), \operatorname{Cos}(G, H, H g H) \cong \operatorname{Cos}\left(G, H, H g^{\prime} H\right)$ if and only if $g^{\sigma} \in g^{\prime} L$ for some $\sigma \in$ $\mathbf{N}_{\text {Aut }(G)}(H) \cap \mathbf{N}_{\text {Aut }(G)}(L)$.

Proof: For $g, g^{\prime} \in I(G, L)$, if there exists $\sigma \in \mathbf{N}_{\text {Aut }(G)}(H) \cap \mathbf{N}_{\text {Aut }(G)}(L)$ such that $g^{\sigma} \in$ $g^{\prime} L$, then $\operatorname{Cos}(G, H, H g H) \cong \operatorname{Cos}\left(G, H, H g^{\sigma} H\right)=\operatorname{Cos}\left(G, H, H g^{\prime} H\right)$.

Conversely, let $g, g^{\prime} \in I(G, L)$ be such that $\operatorname{Cos}(G, H, H g H) \cong$ $\operatorname{Cos}\left(G, H, H g^{\prime} H\right)$. Then $H \cap H^{g}=L=H \cap H^{g^{\prime}}$. Let $\Gamma=\operatorname{Cos}(G, H, H g H)$, $\Gamma^{\prime}=\operatorname{Cos}\left(G, H, H g^{\prime} H\right), A=$ Aut $\Gamma$ and $B=$ Aut $\Gamma^{\prime}$. Then $A \cong B, G \leq A \cap B$, and $G, A$ and $B$ are primitive permutation groups on $[G: H]$. Let $\varphi$ be an isomorphism from $\Gamma$ to $\Gamma^{\prime}$ such that $H^{\varphi}=H$. Then $\varphi$ is a permutation of $[G: H]$, and $A=\varphi B \varphi^{-1} \geq \varphi G \varphi^{-1} \cong G$. By Lemma 3.3, $\varphi G \varphi^{-1}=G$, that is, $\varphi$ normalises $G$. Since $G$ is primitive, the centraliser $\mathbf{C}_{\mathrm{Sym}(V \Gamma)}(G)=1$, so $\varphi$ may be viewed as an automorphism of $G$.

Label the vertices $H, H g$ of $\Gamma$ as $\alpha, \beta$, respectively, and label the vertex $H g^{\prime}$ as $\beta^{\prime}$. Since $\Gamma$ is $G$-arc transitive, we may assume that $\alpha^{\varphi}=\alpha$, and $\beta^{\varphi}=\beta^{\prime}$, that is, $H^{\varphi}=H$ and $(H g)^{\varphi}=H g^{\prime}$. Also $\varphi^{-1} G_{\beta} \varphi=G_{\beta^{\prime}}$, that is, $\varphi^{-1} H^{g} \varphi=H^{g^{\prime}}$. So $L^{\varphi}=\left(H \cap H^{g}\right)^{\varphi}=H \cap H^{g^{\prime}}=L$. Hence $\varphi \in \mathbf{N}_{\text {Aut }(G)}(H) \cap \mathbf{N}_{\text {Aut }(G)}(L)$.

Further, since $\varphi$ fixes the vertex $\alpha, \varphi$ maps the neighborhood $\Gamma(\alpha)$ to the neighborhood $\Gamma^{\prime}(\alpha)$, Thus $H g^{\varphi} H=(H g H)^{\varphi}=H g^{\prime} H$, so $g^{\varphi} \in H g^{\prime} H$, that is, $g^{\varphi}=h_{1} g^{\prime} h_{2}$ for some elements $h_{1}, h_{2} \in H$. Let $\sigma=\varphi h_{1} \in \operatorname{Aut} \Gamma \leq \operatorname{Aut}(G)\left(h_{1}\right.$ acts on $G$ by conjugation) and write $h=h_{2} h_{1}$. Then $\sigma \in \mathbf{N}_{\text {Aut }(G)}(H)$ and

$$
g^{\sigma}=g^{\varphi h_{1}}=g^{\prime} h,
$$

so $g^{-1} g^{\sigma}=h \in H$. Further, it is easily shown that

$$
L^{\sigma}=\left(H \cap H^{g}\right)^{\sigma}=H^{\sigma} \cap\left(g^{-1} H g\right)^{\sigma}=H \cap H^{g^{\prime}}=L .
$$


So $\sigma \in \mathbf{N}_{\text {Aut }(G)}(L)$ and $g^{\sigma} \in \mathbf{N}_{G}(L)$. Since $g^{\prime} \in \mathbf{N}_{G}(L)$, we have $g^{-1} g^{\sigma} \in \mathbf{N}_{G}(L)$, and as $g^{-1} g^{\sigma}=h \in H$, we obtain $h=g^{-1} g^{\sigma} \in H \cap \mathbf{N}_{G}(L)=\mathbf{N}_{H}(L)=L$, so $g^{\sigma}=g^{\prime} h \in g^{\prime} L$.

Finally, we prove a lemma for determining the number of non-isomorphic vertex primitive 2-arc regular graphs. By Lemma 3.4, we need only to consider those graphs generated by the elements of $I(G, L)$. Let

$$
K=\mathbf{N}_{\text {Aut }(G)}(H) \cap \mathbf{N}_{\text {Aut }(G)}(L), \quad \Sigma=\{\bar{g}=g L \mid g \in I(G, L)\} .
$$

For any $\alpha \in K$ and any $g \in I(G, L)$, the image $g^{\alpha} \in I(G, L)$. Thus $K$ has a natural action on the set $\Sigma$ defined as

$$
\bar{g}^{\sigma}=\overline{g^{\sigma}}, \text { for } \sigma \in K \text { and } \bar{g} \in \Sigma \text {. }
$$

Notice that, by the definition of $I(G, L)$, each element $\bar{g} \in \Sigma$ is an involution of $\mathbf{N}_{G}(L) / L$. Furthermore, we have the following lemma to count our graphs.

Lemma 3.5. Let $(G, H, L)$ be one of the entries in Table 2. Let $n$ be the number of non-isomorphic graphs corresponding to the triple $(G, H, L)$. Then

(1) $n$ equals the number of $K$-orbits in $\Sigma$;

(2) if in addition $G=\operatorname{Aut}(G)$, then $n$ equals the number of involutions in $\mathbf{N}_{G}(L) / L$.

Proof: Let $g, g^{\prime} \in I(G, L)$. If $\operatorname{Cos}(G, H, H g H) \cong \operatorname{Cos}\left(G, H, H g^{\prime} H\right)$, then by Lemma 3.4, there is a $\sigma \in K$ such that $g^{\sigma}=g^{\prime} h$ for some $h \in L$. So $\bar{g}^{\sigma}=\overline{g^{\sigma}}=$ $\overline{g^{\prime} h}=\overline{g^{\prime}}$. Conversely, if $\bar{g}$ and $\overline{g^{\prime}}$ belong to the same $K$-orbit, that is, $\bar{g}^{\sigma}=\overline{g^{\prime}}$ for some $\sigma \in K$, then $g^{\sigma} \in g^{\prime} L$. Thus by Lemma 3.4, $\operatorname{Cos}(G, H, H g H) \cong \operatorname{Cos}\left(G, H, H g^{\prime} H\right)$. Therefore, part (1) is true. If $G=\operatorname{Aut}(G)$, then as $H$ is maximal in $G$, we have $K=$ $\mathbf{N}_{G}(H) \cap \mathbf{N}_{G}(L)=H \cap \mathbf{N}_{G}(L)=\mathbf{N}_{H}(L)=L$. Thus $n=|\Sigma|$, equal to the number of involutions in $\mathbf{N}_{G}(L) / L$.

\section{Proof of Theorem 1.1}

As before, let $\Gamma$ be a non-complete graph of valency at least 5 , and assume that $G \leq$ Aut $\Gamma$ is an almost simple group such that $G$ is primitive on $V \Gamma$ and regular on the set of 2-arcs of $\Gamma$. By Lemmas 3.1 and 3.2, we may assume that $G$ is one of the groups listed in Table 2, $\Gamma=\operatorname{Cos}(G, H, H g H)$ where $H=K: L$ is the corresponding maximal subgroup of $G$ and $g \in I(G, L)$. Let $A=$ Aut $\Gamma$, and let $n(A)$ be the number of non-isomorphic graphs with automorphism group $A$. Note that $\mathbf{N}_{G}(L)$ is a subgroup of some maximal subgroup of $G$ containing $L$. We shall process our proof of Theorem 1.1 by analysing the candidates listed in Table 2 one by one.

Lemma 4.1. If $\operatorname{soc}(G)$ is a sporadic simple group or an exceptional simple group of Lie type, then Theorem 1.1 holds. 
Proof: For $G=J_{1}$, we have $H=7: 6$ or $11: 10$, and $L=Z_{6}$ or $Z_{10}$, respectively. By [1], all cyclic subgroups of order $|L|$ are conjugate, and so $L$ is conjugate to a subgroup of $D_{6} \times D_{10}<G$. It follows that $\mathbf{N}_{G}(L) \cong D_{i} \times Z_{2}$ and $\mathbf{N}_{G}(L) / L \cong Z_{2}$, where $i=6$ or 10 . By Lemma 3.3, we have $G \leq A \leq \operatorname{Aut}\left(J_{1}\right)=J_{1}$, so $A=J_{1}$. Then by Lemma $3.5(2)$, there is exactly one graph arising in each case, with valency 7 or 11 , respectively.

For $G=J_{3} .2$, we have $H=19: 18$ and $L=Z_{18}$. By [1], all cyclic subgroups of order 18 are conjugate. Since $G$ contains only one conjugacy class of maximal subgroups $L_{2}(17) \times Z_{2}$ which contain an element of order 18, there exists a subgroup $M=L_{2}(17) \times Z_{2}$ of $G$ such that $\mathbf{N}_{G}(L) \leq M$, which implies that $\mathbf{N}_{G}(L)=\mathbf{N}_{M}(L)$. Note that $M$ has only one conjugacy class of maximal subgroups $D_{18} \times Z_{2}$ containing elements of order 18. Similarly we deduce that $\mathbf{N}_{G}(L)$ is contained in a maximal subgroup of $M$ isomorphic to $D_{18} \times Z_{2}$. It follows that $\mathbf{N}_{G}(L) \cong D_{18} \times Z_{2}$ and $\mathbf{N}_{G}(L) / L \cong Z_{2}$. By Lemma $3.5(2)$, there exists exactly one graph occurring in this case. By Lemma 3.3, we have $G \leq A \leq \operatorname{Aut}\left(J_{3}\right)=J_{3} .2$, so $A=G=$ $J_{3} .2$.

For $G=O N .2$, we have $H=31: 30$, and $L=Z_{30}$. By [1], all cyclic subgroups of order 30 are conjugate. Since $Z_{2} \times D_{6} \times D_{10}$ contains a cyclic subgroup of order $30 \mathrm{we}$ may assume $L<Z_{2} \times D_{6} \times D_{10}=\mathbf{N}_{G}(L)<Z_{2} \times J_{1}<G$. Hence $\mathbf{N}_{G}(L) / L \cong Z_{2}^{2}$. By Lemma 3.3, we have $A=G$, and by Lemma 3.5 (2), there are exactly 3 graphs in this case, that is, $n(A)=3$.

For $G=J_{4}$, we have $H=29: 28$, and $L=Z_{28}$. It follows from the information given in [1] that all cyclic subgroups of order 28 are conjugate. Since the subgroup $2^{3+12}$. $\left(S_{5} \times L_{3}(2)\right)$ contains a cyclic subgroup of order 28 , we may assume that $L<$ $2^{3+12} .\left(S_{5} \times L_{3}(2)\right)<G$. Now $\mathbf{C}_{G}(L)=L$, and if $a$ is an element of $G$ of order 7 , then $a$ is not conjugate to $a^{-1}$. Thus, $\mathbf{N}_{G}(L)=D_{8} \times\left(Z_{7}: Z_{3}\right)$, and so $\mathbf{N}_{G}(L) / L \cong Z_{6}$. By Lemma 3.5 (2), there exists exactly one graph occurring. Then by Lemma 3.3, the automorphism group $A=G=J_{4}$.

For $G=F i_{24}$, we get $H=29: 28$, and $L \cong Z_{28}$. Let $x$ be an element of $L$ of order 28. Then $x=x_{1} x_{2}$ such that $o\left(x_{1}\right)=4$ and $o\left(x_{2}\right)=7$. By [1], $\left|\mathbf{C}_{G}\left(x_{2}\right)\right|=35280$ or 2058. Since 4 does not divide 2058, we have $\left|\mathbf{C}_{G}\left(x_{2}\right)\right|=35280$, so a Sylow 2subgroup $S$ of $\mathbf{C}_{G}\left(x_{2}\right)$ is of order 16. Hence $\mathbf{N}_{S}\left(\left\langle x_{1}\right\rangle\right)>\left\langle x_{1}\right\rangle$. Let $g \in \mathbf{N}_{S}\left(\left\langle x_{1}\right\rangle\right) \backslash\left\langle x_{1}\right\rangle$. Then $x_{1}^{g} \in\left\langle x_{1}\right\rangle$ and $x_{2}^{g}=x_{2}$, so $x^{g} \in\langle x\rangle$. Hence by Lemma 3.2, there exists at least one graph. By Lemma 3.3, $A=F i_{24}$. Again by [1], $\mathbf{C}_{G}(x)=\langle x\rangle=L$. Thus the number of involutions of $\mathbf{N}_{G}(L) / L$ is not greater than the number of involutions of $\operatorname{Aut}(L) \cong Z_{2} \times Z_{6}$. Thus there are one or three graphs in this case, that is, $n(A)=1$ or 3 .

For the Monster simple group $G=M$, we have $H=41: 40$, and $H=Z_{40}$. By Lemma 3.3, if there exists a graph $\Gamma$ in this case, the full automorphism group $A=M$. By [1], we conclude that $\left|\mathbf{C}_{G}\left(Z_{40}\right) / Z_{40}\right|=10,8$ or 2 . Thus there exists at least one graph, that is, $n(A) \geq 1$.

For the Suzuki group $G=S z(8)$, we get $H=5: 4$, and $L=Z_{4}$. Let $x$ be an element of $L$ of order 4. By [1], $x$ is not conjugate to its inverse and $\mathbf{N}_{G}(L)=\mathbf{C}_{G}(L)$ has size 16. Let $Q$ be a Sylow 2-subgroup of $G$ containing $L=\langle x\rangle$. By Lemma 1 of [18], we have that $Z(Q)=Z_{2}^{3}$. Thus $\mathbf{N}_{G}(L)$ contains a subgroup $Z_{2}^{3}$, and so $\mathbf{N}_{G}(L)$ is not isomorphic to $Z_{4}^{2}$. It follows that $\mathbf{N}_{G}(L)=\mathbf{C}_{G}(L) \cong Z_{4} \times Z_{2}^{2}$. Now $F=\mathbf{N}_{\text {Aut }(G)}(H) \cap$ $\mathbf{N}_{\text {Aut }(G)}(L)=Z_{4} \times Z_{3}$. Further, $Z_{3} \cong \operatorname{Out}(G)$ fixes $x^{2}$ and is semiregular on the set of 
the other 6 involutions of $\mathbf{N}_{G}(L)$. Since $\operatorname{Cos}(G, H, H g H)=\operatorname{Cos}\left(G, H, H g x^{2} H\right)$, it follows from Lemma 3.5 that exactly one graph occurs in this case, so that $n(A)=1$. By Lemma 3.3, it is easily shown that $A=G=S z(8)$.

For $G=S z(8) .3$, the subgroups $H=7: 6$ and $L=Z_{6}$. By the information given in [1], we obtain $\mathbf{N}_{G}(L) / L \cong Z_{2}$. Thus by Lemma 3.5 , there is exactly one graph in this case. Since $G=\operatorname{Aut}(G)$, we have Aut $\Gamma=G$ by Lemma 3.3.

For the case $G={ }^{3} D_{4}(2) .3$, the subgroups $H=13: 12$ and $L=Z_{12}$. Let $x$ be an element of $L$ of order 12. Then $x=x_{1} x_{2}$ such that $o\left(x_{1}\right)=4$ and $o\left(x_{2}\right)=3$. By [1], we get $\left|\mathbf{C}_{G}\left(x_{2}\right)\right|=12096$ or 216 . Thus a Sylow 2-subgroup $S$ of $\mathbf{C}_{G}\left(x_{2}\right)$ is of order 8 , and so $\mathbf{N}_{S}\left(\left\langle x_{1}\right\rangle\right)>\left\langle x_{1}\right\rangle$. Let $g \in \mathbf{N}_{S}\left(\left\langle x_{1}\right\rangle\right) \backslash\left\langle x_{1}\right\rangle$. Then $x_{1}^{g} \in\left\langle x_{1}\right\rangle$, and $x_{2}^{g}=x_{2}$. So $x^{g}=x_{1}^{g} x_{2}^{g} \in\langle x\rangle=L$, and $L^{g}=L$. Since $\operatorname{Aut}(G)=G$, by Lemma 3.3, if there exists a graph for this case, the automorphism group $A=G$. Again by [1], $\mathbf{C}_{G}(L)=L$. Thus the number of involutions of $\mathbf{N}_{G}(L) / L$ is not greater than the number of involutions of Aut $(G) \cong Z_{2} \times Z_{2}$. By Lemma 3.5 (2), exactly 1 or 3 graphs occur in the case, that is, $n(A)=1$ or 3 .

For $G={ }^{2} F_{4}(2)$, we have $H=13: 12$, and $L=Z_{12}$. It follows from the information given in the Atlas [1] that all cyclic subgroups of $G$ of order 12 are conjugate, and so $L<L_{2}(25)$.2. Further analysis gives $\mathbf{N}_{G}(L)=\mathrm{D}_{24}$, and so $\left|\mathbf{N}_{G}(L) / L\right|=2$. By Lemma $3.5(2)$, there exists exactly one graph for this case. Since $\operatorname{Aut}(G)=G$, we have $A=G$ by Lemma 3.3 .

For $G={ }^{2} F_{4}(8) .3$, we have $H=37: 36$ and $L=Z_{36}$. If $\Gamma$ exists, its valency is 37, and since $\operatorname{Aut}(G)=G$, we have $A=G$ by Lemma 3.3. Let $\sigma$ be an automorphism of $T:={ }^{2} F_{4}(8)$ induced by a field automorphism of order 3 . Then $G={ }^{2} F_{4}(8) .\langle\sigma\rangle$. Write $L=\left\langle x_{0}\right\rangle$ and $x_{0}=x_{1} x_{2}$ such that $o\left(x_{1}\right)=9$ and $o\left(x_{2}\right)=4$. Let $x=x_{1}^{3}$. Then $x \in T$ is of order 3. By [17] (or see the first four lines of [12, 1.2]), all elements of $T$ of order 3 are conjugate. By Proposition 1.2 and its corollary of [12], we have

$$
\langle x\rangle \times U_{3}(8) .2=\mathbf{C}_{T}(x)<C_{G}(x)=\left(\langle x\rangle \times U_{3}(8) \cdot 2\right) \cdot\langle\sigma\rangle .
$$

Now $L \leq \mathbf{C}_{G}(x)<\left(\langle x\rangle \times U_{3}(8) .2\right)$. $\langle\sigma\rangle$. Since $\mathbf{C}_{G}(L) \leq \mathbf{C}_{G}(x)$, we obtain

$$
x_{2} \in L \triangleleft \mathbf{C}_{G}(L) \leq \mathbf{C}_{G}(x)=\left(\langle x\rangle \times U_{3}(8) .2\right) .\langle\sigma\rangle .
$$

Let $S$ be a Sylow 2-subgroup of $U_{3}(8) .2$ containing $x_{2}$. Then by the Atlas [1], $S=$ $2^{3+6}: 2$ and $S^{x_{1}}=S$. Let

$$
C=\mathbf{C}_{S}\left(x_{2}\right), \quad N=\mathbf{N}_{S}\left(\left\langle x_{2}\right\rangle\right)
$$

Since $C^{x_{1}}$ centralises $x_{2}^{x_{1}}=x_{2}$, we have $C^{x_{1}}=C$. Further, for $u \in N, C^{u^{x_{1}}}=C$ and hence $N^{x_{1}}=N$. Let $\Omega=\mathbf{N}_{S}\left(\left\langle x_{2}\right\rangle\right) \backslash\left\langle x_{2}\right\rangle$. Assume first that $x_{2} \in U_{3}(8)$. Using GAP, computation shows that $|C|=2^{6}$ and $|N|=2^{7}$. Then $|\Omega|=2^{7}-4=124$, not divisible by 3 . Since $x_{1}$ normalises both $\left\langle x_{2}\right\rangle$ and $N$, we have that $x_{1}$ fixes $\Omega$ setwise (by conjugation) and centralises at least one element of $\Omega$. Choose $g$ to be such an element such that $g^{2} \in L$. Then $g$ normalises $L$, and $g \in I(G, L)$. Assume now that $x_{2} \in U_{3}(8) .2 \backslash U_{3}(8)$. Using GAP, computation shows that $|C|=2^{7}=128$ and $|N|=2^{8}=256$. Since $x_{1}$ normalises $C$ and $\left|C \backslash\left\langle x_{2}\right\rangle\right|=124$. Arguing as in the 
previous case, we get a 2 -element $g \in C \backslash\left\langle x_{2}\right\rangle \subset \Omega$, which lies in $I(G, L)$. Thus by Lemma 3.2, there exists a graph $\Gamma$.

Finally, for the Ree group $G={ }^{2} G_{2}(27) .3$, we have $H=19: 18$ and $L=Z_{18}$. From the information given in the Atlas [1], it is easily shown that there is no 2-element satisfying Lemma 2.3. So no graph occurs for this case.

Lemma 4.2. If the socle of $G$ is $A_{n}$ for some $n \geq 5$, then Theorem 1.1 holds.

Proof: For $G=A_{6} .2=M_{10}$, we have $H=5: 4$ and $L=Z_{4}$. It follows from [1] that $\mathbf{N}_{G}(L)=Q_{8}$, and so $I(G, L) \subseteq Q_{8} \backslash L$. On the other hand, since $H=\mathbf{N}_{G}(H)=K: L$, we conclude that $\left(Q_{8} \backslash L\right) \cap \mathbf{N}_{G}(H)=\emptyset$. Thus $I(G, L)=Q_{8} \backslash L$. Since $\left|Q_{8}: L\right|=2$, we have that $L x=L y$ for any $x, y \in Q_{8} \backslash L=I(G, L)$. Hence $|\Sigma|=1$, and $\operatorname{son}(A)=$ 1. By [1], $\mathbf{N}_{\text {Aut }(G)}(H)=H \times Z_{2}$, so $\operatorname{Aut} \Gamma=\operatorname{Aut}(G)$.

If $G=S_{p}$ then $H=Z_{p}: Z_{p-1}$. It is easy to show that $\mathbf{C}_{G}\left(Z_{p-1}\right)=Z_{p-1}$ and $\operatorname{Aut}\left(Z_{p-1}\right)<S_{p}$. Hence $\mathbf{N}_{G}\left(Z_{p-1}\right) / Z_{p-1}=\operatorname{Aut}\left(Z_{p-1}\right)$, and by Lemma $3.5(2), n(A)$ equals the number of involutions of $\mathbf{N}_{G}\left(Z_{p-1}\right) / Z_{p-1}=\operatorname{Aut}\left(Z_{p-1}\right)$. Let

$p-1=p_{1}^{r_{1}} p_{2}^{r_{2}} \ldots p_{k}^{r_{k}}, \quad$ where $p_{1}=2$ and $p_{i}$ are distinct odd primes for $i \geq 2$.

Then $\operatorname{Aut}\left(Z_{p-1}\right)=\operatorname{Aut}\left(Z_{2^{r_{1}}}\right) \times \operatorname{Aut}\left(Z_{p_{2}^{r_{2}}}\right) \times \cdots \times \operatorname{Aut}\left(Z_{p_{k}^{r_{k}}}\right)$. A Sylow 2-subgroup $T$ of $\operatorname{Aut}\left(Z_{p-1}\right)$ is isomorphic to $\operatorname{Aut}\left(Z_{2^{r_{1}}}\right) \times Z_{2^{s_{2}}} \times \cdots \times Z_{2^{s_{k}}}$, where $p_{i} \equiv 1\left(\bmod 2^{s_{i}}\right)$. Note that $T$ is normal in $\operatorname{Aut}\left(Z_{p-1}\right)$ and $\operatorname{Aut}\left(Z_{2^{r_{1}}}\right)=1, Z_{2}$ or $Z_{2} \times Z_{2^{r_{1}-2}}$ depending on $r_{1}=1,2$ or $\geq 3$. All involutions of $\mathbf{N}_{G}\left(Z_{p-1}\right) / Z_{p-1}$ are in $T$. Thus $n(A)=2^{k-1}-1$, $2^{k}-1$ or $2^{k+1}-1$. Since $\operatorname{Aut}(G)=G$, we have Aut $\Gamma=G$.

Lemma 4.3. If $G=L_{3}(4), S_{4}(4) .4$, or $U_{3}(4) .4$, then Theorem 1.1 holds.

Proof: For $G=L_{3}(4)$, we have $H=3^{2}: Q_{8}$ and $L=Q_{8}$. Using GAP, computation shows that $G$ has a unique self-paired 2-transitive subconstituent of length 9 . Thus $n(A)=1$. By the information given in [1], $\mathbf{N}_{\mathrm{Aut}(G)}(H)=H . D_{12}$, and so Aut $\Gamma=G . D_{12}$.

For $G=S_{4}(4) .4$, we have $H=17: 16$ and $L=Z_{16}$. Using GAP, computation shows that $G$ has a unique self-paired 2-transitive subconstituent of length 17 . Thus $n(A)=1$. Since $G=\operatorname{Aut}(G)$, by Lemma 3.3, Aut $\Gamma=G$.

Finally, for $G=U_{3}(4) .4$, we have $H=13: 12$ and $L=Z_{12}$. Let $x$ be an element of $L$ of order 12, and $x=x_{1} x_{2}$ such that $o\left(x_{1}\right)=3$ and $o\left(x_{2}\right)=4$. By [1], $\mathbf{C}_{G}(L)=L$. Thus $\mathbf{N}_{G}(L) / L \leq \operatorname{Aut}(L) \cong Z_{4}$. So $\left|\mathbf{N}_{G}(L) / L\right|=1,2$ or 4 . Again by [1] we know that all cyclic subgroups of $G$ of order 12 are conjugate in $G$ and that there is a maximal subgroup $5^{2}:\left(4 \times S_{3}\right)$. So we may assume that $L<4 \times S_{3}$, which implies that $\left|\mathbf{N}_{G}(L) / L\right| \neq 1$. If $\left|\mathbf{N}_{G}(L) / L\right|=4$, then the order of a Sylow 2-subgroup of $\mathbf{N}_{G}(L)$ is 16. Let $T$ be a Sylow 2-subgroup of $\mathbf{N}_{G}(L)$. It follows that $L<T$. Moreover there are two distinct two elements $x, y \in \mathbf{N}_{G}(L) \backslash L$ such that $T=\left\langle x_{2}, x, y\right\rangle$. Since $\left\langle x_{1}\right\rangle$ is a characteristic subgroup of $L,\left\langle x_{1}\right\rangle^{x}=\left\langle x_{1}\right\rangle^{y}=\left\langle x_{1}\right\rangle$. On the other hand from [1] we know that the order of a Sylow 2-subgroup of $\mathbf{C}_{G}\left(\left\langle x_{1}\right\rangle\right)$ is 4 and hence $\left\langle x_{2}\right\rangle$ is the Sylow 2-subgroup of $\mathbf{C}_{G}\left(\left\langle x_{1}\right\rangle\right)$, which implies that $x_{1}^{x}=x_{1}^{y}=x_{1}^{-1}$. Thus $x_{1}^{x y^{-1}}=x_{1}$ and hence $x y^{-1} \in \mathbf{C}_{G}\left(\left\langle x_{1}\right\rangle\right) \cap T=\left\langle x_{2}\right\rangle<L$. Thus $T=\langle L, x\rangle$ and $x^{2} \notin L$ (for otherwise $|T| \neq 16)$. However $x_{1}^{x^{2}}=x_{1}$ and hence $x^{2} \in \mathbf{C}_{G}\left(\left\langle x_{1}\right\rangle\right) \cap T=\left\langle x_{2}\right\rangle<L$, which is a 
contradiction. Thus $\left|\mathbf{N}_{G}(L) / L\right|=2$ and $n(A)=1$ as expected. Since $G=\operatorname{Aut}(G)$, Aut $\Gamma=G$.

Lemma 4.4. Let $G=U_{3}\left(2^{r}\right)$ and $H=Z_{3}^{2}: Q_{8}$, where $r>3$ is a prime. Then Aut $\Gamma=$ $U_{3}\left(2^{r}\right) . S_{3}$, and the number of non-isomorphic graphs equals $\left(2^{r-1}-1\right) / r$.

Proof: Now $L \cong Q_{8}$. First we determine $I(G, L)$. It follows from [5, XI 13.9] that the intersection of any two Sylow 2-subgroups is equal to the identity group. Thus $L$ is contained in a unique Sylow 2-subgroup $S$. If $g \in I(G, L)$, then $\langle L, g\rangle \leq S$ and so $I(G, L) \leq S$. By [5, VIII 7.10], $S$ is a Suzuki 2-group with $|S|=|\mathbf{Z}(S)|^{3}$. By [5, VIII 7.9], $S^{\prime}=\mathbf{Z}(S)$ consists of the identity and all involutions of $S$. It is clear that $\mathbf{Z}(S) \leq \mathbf{N}_{S}(L)$ and $L \mathbf{Z}(S) \backslash L \leq I(G, L)$. Conversely, assume that $g \in I(G, L)$. If $g$ is an involution, then since $g \in S, g \in \mathbf{Z}(S) \subset L \mathbf{Z}(S)$. If $o(g)=4$, then $g^{2}$ is the unique involution of $L$. Suppose that $L=\langle a, b\rangle$ with $o(a)=4=o(b)$. Assume that $g \notin L \mathbf{Z}(S)$. Since $\langle a, b\rangle^{g}=\langle a, b\rangle$ and $S^{\prime}=\mathbf{Z}(S), a^{g}=g^{-1} a g a^{-1} a=e a$ where $e=g^{-1} a g a^{-1} \in \mathbf{Z}(S)$. Since $g^{-1} a g, a^{-1} \in L$, we have $e \in L$ and so $e=1$ or $a^{2}$. Thus $a^{g}=a$ or $a^{-1}$. If $a^{g}=a$ then $\langle a, g\rangle$ is an abelian group and contains the unique involution $a^{2}=g^{2}$. By [4, III 8.2], $\langle a, g\rangle$ is cyclic and so $g \in\langle a\rangle$ as $\exp (S)=4$, a contradiction. Thus $a^{g}=a^{-1}$. Similarly $b^{g}=b^{-1}$. It follows that $\langle a, b, g\rangle$ contains the unique involution $a^{2}=b^{2}=g^{2}$. Again by [4, III 8.2], $\langle a, b, g\rangle \cong Q_{8}$ as $\exp (S)=4$, so $g \in\langle a, b\rangle$, a contradiction. Thus we conclude that $g \in L \mathbf{Z}(S)$ and $I(G, L)=L \mathbf{Z}(S) \backslash L$. By Lemma 3.2, all 2-arc transitive graphs obtained from $G$ satisfying our requirement are determined by $\mathbf{Z}(S) \backslash\left\{1, a^{2}\right\}$. Now we consider the action of $F:=\mathbf{N}_{\text {Aut }(G)}(H) \cap \mathbf{N}_{\text {Aut }(G)}(L)$ on $\mathbf{Z}(S)$. In the meaning of Aschbacher's eight subgroup collections (see [7, page 71, Table 3.5.B]), $H=3^{2}: Q_{8}$ is a member of $\mathcal{C}_{5}$. Hence $H=U_{3}(2)$. It is clear that $U_{3}(2) \triangleleft P G U_{3}(2)<P G U_{3}\left(2^{r}\right)$, which implies that the diagonal automorphism of $G$ normalizes $H$. Furthermore, the field automorphism of $G$ induces an automorphism of order 2 on the ground field $G F\left(2^{2}\right)$ on which $H=U_{3}(2)$ is defined. Hence the field automorphism of $G$ normalizes $H$. It follows that $\mathbf{N}_{\text {Aut }(G)}(H)=H:\left(Z_{3}: Z_{2 r}\right)=H:\left(S_{3} \times Z_{r}\right)=Z_{3}^{2}:\left(L:\left(S_{3} \times Z_{r}\right)\right)$. Thus $F=L:\left(S_{3} \times\right.$ $\left.Z_{r}\right)=\left(L: S_{3}\right) \times Z_{r}$. It is clear that $\mathbf{N}_{G}(\mathbf{Z}(S)) \geq \mathbf{N}_{G}(S)$. By [4, II 10.12], $\mathbf{N}_{G}(S)$ is maximal in $G$, so $\mathbf{N}_{G}(\mathbf{Z}(S))=\mathbf{N}_{G}(S)=S: Z_{\left(2^{2 r}-1\right) / 3}$. Furthermore, it is not hard to verify that $\mathbf{C}_{G}(\mathbf{Z}(S))=S: Z_{\left(2^{r}+1\right) / 3}$. Hence $\mathbf{N}_{G}(\mathbf{Z}(S)) / \mathbf{C}_{G}(\mathbf{Z}(S)) \cong Z_{2^{r}-1}$. Similarly one can prove that $\mathbf{N}_{\text {Aut }(G)}(\mathbf{Z}(S))=S:\left(Z_{2^{2 r}-1}: Z_{2 r}\right)$ and $\mathbf{C}_{\text {Aut }(G)}(\mathbf{Z}(S))=S:\left(Z_{2^{r}+1}: Z_{2}\right)$, which implies that $\mathbf{N}_{\text {Aut }(G)}(\mathbf{Z}(S)) / \mathbf{C}_{\text {Aut }(G)}(\mathbf{Z}(S)) \cong Z_{2^{r-1}}: Z_{r}$. Thus the kernel of $F$ acting on $\mathbf{Z}(S)$ is equal to $L: S_{3}$. So $F$ fixes $a^{2}$ and acts semiregularly on the other involutions of $\mathbf{Z}(S)$, namely, on $\mathbf{Z}(S) \backslash\left\{1, a^{2}\right\}$. Since $\operatorname{Cos}(G, H, H g H)=\operatorname{Cos}\left(G, H, H g a^{2} H\right)$, it follows that $n(A)=\left(\frac{2^{r}-2}{2}\right) / r=\left(2^{r-1}-1\right) / r$. Since $S_{3}$ normalises $H$ and fixes every involution of $\mathbf{Z}(S)$, we have Aut $\Gamma=G . S_{3}$.

Lemma 4.5. Let $G=L_{3}(q)$, where $q \equiv 4,7(\bmod 9)$ is a prime, and $H=Z_{3}^{2}: Q_{8}$. Then

$$
(\text { Aut } \Gamma, n)= \begin{cases}(G, 1), & \text { if } q \equiv 3(\bmod 4), \\ (G, 2) \text { or }(\operatorname{Aut}(G), 1), & \text { if } q \equiv 1(\bmod 4),\end{cases}
$$

where $n$ is the number of non-isomorphic corresponding graphs. 
Proof: All subgroups of $L_{3}(q)$ for odd $q$ were determined by Mitchell [13]. Let $L=Q_{8}<H$. It is to show that 16 divides $|G|$ and so $\mathbf{N}_{G}(L)>L$. By checking the subgroups determined in [13], we get $\mathbf{N}_{G}(L)=Z_{(q-1) / 3} . S_{4}$ and $\left[Z_{(q-1) / 3}, S_{4}\right]=1$. Let $M$ be a Hall $2^{\prime}$-subgroup and $\langle x\rangle$ a Sylow 2-subgroup of $Z_{(q-\varepsilon) / 2}$. Since $q \equiv 4,7(\bmod$ 9), 3 does not divide $(q-\varepsilon) / 3$. It follows that $\mathbf{N}_{G}(L)=M \times\left(\langle a\rangle . S_{4}\right)$. Furthermore, $\langle a\rangle . S_{4}=\langle a\rangle \circ(L .\langle x, y\rangle)=(\langle a\rangle \circ L) .\langle x, y\rangle$, where $\langle a\rangle \cap L=Z_{2}, o(x)=3,\langle x, y\rangle=$ $S_{3}$ and $L .\langle x, y\rangle=2 S_{4}$. It is not hard to write out all elements of $I(G, L)$ :

$$
I(G, L)= \begin{cases}\left\{y, y^{x}, y^{x^{2}}\right\}^{L} & \text { if } o(a)=2 \\ \left\{y, y^{x}, y^{x^{2}}, b, b y, b y^{x}, b y^{x^{2}}\right\}^{L} & \text { if } o(a) \geq 4\end{cases}
$$

where $b$ is an element of $\langle a\rangle$ of order 4. Again by [11], we have $\mathbf{N}_{\text {Aut }(G)}(H)=$ $\left(3^{2}: L\right) . O=3^{2}:(L . O)$, where $O=S_{3}$ and $L . O=2 S_{4}$, and $\mathbf{N}_{\text {Aut }(G)}(L)=Z_{q-1} \cdot S_{4} \cdot\langle v\rangle$ such that its center is $Z_{q-1}$, where $v$ is a graph automorphism of $G$ of order 2 . Thus $O<$ $\mathbf{N}_{\text {Aut }(G)}(L)$, no element of $O$ centralizes $L$ and $O \cap G=1 ; Z_{q-1}=M \times\langle a\rangle \times\langle u\rangle$, where $\langle u\rangle=Z_{3}$ and $\langle u, v\rangle=S_{3}$. So $F:=\mathbf{N}_{\text {Aut }(G)}(H) \cap \mathbf{N}_{\operatorname{Aut}(G)}(L)=L$.O. Since Aut $\left(S_{4}\right)=S_{4}, v$ acts on $S_{4}$ trivially. It follows that

$$
\begin{aligned}
\mathbf{N}_{\text {Aut }(G)}(L) & =(M \times\langle a\rangle \times\langle u\rangle) .\left(S_{4} \times\langle v\rangle\right) \\
& =M \times(\langle u\rangle \times((\langle a\rangle \circ L) .\langle x, y\rangle)) .\langle v\rangle \\
& =M \times\langle u, v\rangle \times((\langle a\rangle \circ L) .\langle x, y\rangle) .
\end{aligned}
$$

Since $S_{3}=O<\mathbf{N}_{\mathrm{Aut}(G)}(L)$, no element of $O$ centralizes $L$, we have $O=\left\langle u x^{l}, v y^{l}\right\rangle$ where $l \in L$. We need to know the action of $F=L . O$ on $I(G, L)$. To do this, we need only consider the action of $O^{l^{-1}}=\langle u x, v y\rangle$ on $I(G, L)$. First suppose $o(a) \geq 4$. Then

$$
\left\{\begin{array}{l}
y^{u x}=y^{x}, \quad\left(y^{x}\right)^{u x}=y^{x^{2}}, \quad\left(y^{x^{2}}\right)^{u x}=y ; \\
y^{v y}=y, \quad\left(y^{x}\right)^{v y}=y^{v x y}=y^{x y}=y^{y x y}=y^{x^{2}}, \quad\left(y^{x^{2}}\right)^{v y}=y^{x} \\
b^{u x}=b, \quad b^{v y}=b ; \\
(b y)^{u x}=b y^{x}, \quad\left(b y^{x}\right)^{u x}=b y^{x^{2}}, \quad\left(b y^{x^{2}}\right)^{u x}=b y \\
(b y)^{v y}=b y, \quad\left(b y^{x}\right)^{v y}=b y^{x}, \quad\left(b y^{x^{2}}\right)^{v y}=b y^{x^{2}}
\end{array}\right.
$$

It follows from Lemma 3.5 that $n(A)=3$, that there are exactly three non-isomorphic graphs, which are generated by $y, b$ and $b y$ respectively. By Lemma 3.3, if $\Gamma=\operatorname{Cos}(G, H, H y H)$ or $\operatorname{Cos}(G, H, H b y H)$ then $A u t \Gamma=G$, so $n(G)=2$; if $\Gamma=$ $\operatorname{Cos}(G, H, H b H)$ then $\operatorname{Aut} \Gamma=G . S_{3}=\operatorname{Aut}(G)$, so $n(\operatorname{Aut}(G))=1$. If $o(a)=2$, the same argument leads to $n(A)=1$ and $A=A$ ut $\Gamma=G$. Note that $o(a) \geq 4$ if and only if 4 divides $q-1$. This completes the proof of the lemma. 
Lemma 4.6. Let $G=U_{3}(q)$, where $q \equiv-4,-7(\bmod 9)$ is a prime, and $H=Z_{3}^{2}: Q_{8}$. Then

$$
\text { (Aut } \Gamma, n)= \begin{cases}(G .2,1), & \text { if } q \equiv 3(\bmod 4) \\ (G .2,2) \operatorname{or}(G .6,1), & \text { if } q \equiv 1(\bmod 4)\end{cases}
$$

Proof: The techniques and organization of the proof are similar to that of Lemma 4.5. All subgroups of $U_{3}(q)$ for odd $q$ were determined by Mitchell [13]. Let $L=Q_{8}<$ $H$. Clearly, 16 divides $|G|$, so $\mathbf{N}_{G}(L)>L$. By checking the subgroups determined in [13], we get $\mathbf{N}_{G}(L)=Z_{(q-1) / 3} \cdot S_{4}$ and $\left[Z_{(q-1) / 3}, S_{4}\right]=1$. Similar to the proof of Lemma 4.5, $\mathbf{N}_{G}(L)=(M \times\langle a\rangle) \cdot\left(Z_{2}^{2}:\langle x, y\rangle\right)=M \times((\langle a\rangle \circ L) \cdot\langle x, y\rangle)$, where $M$ is a Hall $2^{\prime}$-subgroup of $Z_{(q-1) / 3},\langle a\rangle$ is a Sylow 2-subgroup of $Z_{(q-1) / 3},\langle a\rangle \cap L=Z_{2}$, $o(x)=3,\langle x, y\rangle=S_{3}$ and $L .\langle x, y\rangle=2 S_{4}$. It is not hard to write out all elements of $I(G, L)$ :

$$
I(G, L)= \begin{cases}\left\{y, y^{x}, y^{x^{2}}\right\}^{L} & \text { if } o(a)=2 \\ \left\{y, y^{x}, y^{x^{2}}, b, b y, b y^{x}, b y^{x^{2}}\right\}^{L} & \text { if } o(a) \geq 4\end{cases}
$$

where $b$ is an element of $\langle a\rangle$ of order 4. Again by [11], we have $\mathbf{N}_{\text {Aut }(G)}(H)=\left(3^{2}: L\right) . O$, where $O=Z_{6}$ and $L . O=2 A_{4} \times Z_{2}$, and $\mathbf{N}_{\text {Aut }(G)}(L)=Z_{q-1} . S_{4} \cdot\langle v\rangle$ such that its center is $Z_{q-1} \times Z_{2}$ and $\langle v\rangle=Z_{2}$. Thus $O<\mathbf{N}_{\text {Aut }(G)}(L)$, no element of order 3 of $O$ centralizes $L$ and $O \cap G=1 ; Z_{q-1}=M \times\langle a\rangle \times\langle u\rangle$, where $\langle u\rangle=Z_{3}$ and $\langle u, v\rangle=Z_{6}$. Since $S_{4}$ is a complete group, the out-automorphism $v$ of $G$ of order 2 acts trivially on $S_{4}$. It follows that

$$
\begin{aligned}
\mathbf{N}_{\text {Aut }(G)}(L) & =(M \times\langle a\rangle \times\langle u\rangle) .\left(S_{4} \times\langle v\rangle\right) \\
& =M \times(\langle u\rangle \times((\langle a\rangle \circ L) \cdot\langle x, y\rangle)) .\langle v\rangle \\
& =M \times\langle u, v\rangle \times((\langle a\rangle \circ L) .\langle x, y\rangle) .
\end{aligned}
$$

Now $O=Z_{6}$ is a subgroup of $\mathbf{N}_{\text {Aut }(G)}(L)$. It follows that $O=\left\langle u x^{l}, v\right\rangle$ where $l \in L$. We need to know the action of $\mathbf{N}_{\text {Aut }(G)}(H) \cap \mathbf{N}_{\text {Aut }(G)}(L)=L . O$ on $I(G, L)$. Clearly we need only consider the action of $O^{l^{-1}}=\langle u x, v\rangle$ on $I(G, L)$. First suppose $o(a) \geq 4$. Then

$$
\left\{\begin{array}{l}
y^{u x}=y^{x}, \quad\left(y^{x}\right)^{u x}=y^{x^{2}}, \quad\left(y^{x^{2}}\right)^{u x}=y ; \\
y^{v}=y, \quad\left(y^{x}\right)^{v}=y^{v x}=y^{x},\left(y^{x^{2}}\right)^{v}=y^{x^{2}} ; \\
b^{u x}=b, \quad b^{v}=b ; \\
(b y)^{u x}=b y^{x}, \quad\left(b y^{x}\right)^{u x}=b y^{x^{2}}, \quad\left(b y^{x^{2}}\right)^{u x}=b y ; \\
(b y)^{v}=b y, \quad\left(b y^{x}\right)^{v}=b y^{x}, \quad\left(b y^{x^{2}}\right)^{v}=b y^{x^{2}} .
\end{array}\right.
$$

It follows from Lemma 3.5 that $n(A)=3$, that there are exactly three non-isomorphic graphs, which are generated by $y, b$ and $b y$. By Lemma 3.3, if $\Gamma=\operatorname{Cos}(G, H, H y H)$ or $\operatorname{Cos}(G, H, H b y H)$ then $\operatorname{Aut} \Gamma=G .2$, so $n(G .2)=2$; if $\Gamma=\operatorname{Cos}(G, H, H b H)$ then 基Springer 
Aut $\Gamma=G \cdot Z_{6}=\operatorname{Aut}(G)$, so $n(\operatorname{Aut}(G))=1$. If $o(a)=2$, the same argument leads to $n(A)=1$ and Aut $\Gamma=G$. Note that $o(a) \geq 4$ if and only if 4 divides $q-1$. The lemma is now proved.

Finally, we summarize the arguments for proving Theorem 1.1.

Proof of Theorem 1.1: Let $\Gamma$ be a graph, and assume that $G \leq$ Aut $\Gamma$ acts primitively on the vertex set $V \Gamma$ and regularly on the set of 2 -arcs of $\Gamma$.

By Lemma 2.1, there are three cases that we need to deal with, that is, $\Gamma$ is a complete graph, $G$ is affine type, and $G$ is almost simple. By Lemma 2.2, the first case is as in Theorem 1.1(1), and the second case is as in Theorem 1.1 (2). Thus we only need to consider the case where $G$ is an almost simple group.

By Lemma 3.1, if the valency of $\Gamma$ is at most 4, then Theorem 1.1 holds by the result of [9]. Thus assume that the valency of $\Gamma$ is at least 5 . Then all candidates for $G$ are listed in Table 2, see Lemma 3.1. So by Lemmas 4.1-4.6, for all the possibilities, Theorem 1.1 holds. This completes the proof of Theorem 1.1.

Acknowledgements The second author is supported by a QEII Fellowship of the Australian Research Council. The first and third authors are supported by the National Natural Science Foundation of China and RFDP of China.

The authors are grateful to the referees for their constructive suggestions.

\section{References}

1. J.H. Conway, R.T. Curtis, S.P. Noton, R.A. Parker, and R.A. Wilson, Atlas of Finite Groups, Clarendon Press, Oxford, 1985.

2. X.G. Fang and C.E. Praeger, "Two-arc transitive graphs admitting an almost simple group with socle Suzuki group," Comm. Algebra 27(8) (1999), 3727-3754.

3. The GAP Group, GAP_Groups, Algorithms, and Programming, Version 4.4; 2005, (fttp://www.gapsystem.org).

4. B. Huppert, Endliche Gruppen I, Springer-Verlag, Berlin, 1968.

5. B. Huppert and N. Blackburn, Finite Groups II, III, Springer-Verlag, Berlin, 1982.

6. A.A. Ivanov and C.E. Praeger, "On finite affine 2-arc transitive graphs," European J. Combin. 14 (1993), 421-444.

7. P. Kleidman and M. Liebeck, The Subgroup Structure of The Finite Classical Groups, Cambridge University Press, New York, Sydney, 1990.

8. C.H. Li, "The finite vertex-primitive and vertex-biprimitive $s$-transitive graphs for $s \geq 4$," Trans. Amer. Math. Soc. 353(9) (2001), 3511-3529.

9. C.H. Li, Z.P. Lu and D. Marusic, "On primitive permutation groups with small suborbits and their orbital graphs," J. Algebra 279(2) (2004), 749-770.

10. C.H. Li and A. Seress, "Symmetrical path-cycle covers of a graph and polygonal graphs," J. Combin. Theory Ser. A, (to appear); (electronically available online 10 March 2006).

11. M.W. Liebeck, C.E. Praeger and J. Saxl, "A classification of the maximal subgroups of the finite alternating and symmetric groups," J. Algebra 111 (1987), 365-383.

12. G. Malle, "The maximal subgroups of ${ }^{2} F_{4}\left(q^{2}\right)$," J. Algebra 139 (1991), 52-69.

13. H.H. Mitchell, "Determination of the ordinary and modular ternary linear groups," Trans. Amer. Math. Soc. 12 (1911), 207-242.

14. M. Perkel, "Near-polygonal graphs," Ars. Comb. 26A (1988), 149-170.

15. M. Perkel and C.E. Praeger, "Polygonal graphs: new families and an approach to their analysis," Conr. Num. 124 (1997), 161-173.

16. C.E. Praeger, “An O’Nan-Scott theorem for finite quasiprimitive permutation groups and an application to 2-arc transitive graphs," J. London Math. Soc. 47(2) (1993) 227-239. 
17. K. Shinoda, "A characterization of odd order extensions of Ree groups of type $\left(F_{4}\right)$," J. Fac. Sci. Univ. Tokyo 22 (1975), 79-102.

18. M. Suzuki, "On a class of doubly transitive groups," Ann. Math. 75 (1962), 105-145.

19. W.T. Tutte, "A family of cubical graphs," Proc. Cambridge Phil. Soc. 43 (1947), 459-474.

20. J. Wang, "Primitive permutation groups with a sharply 2-transitive subconstituent," J. Algebra 176 (1995), 702-734.

21. R.M. Weiss, "The nonexistence of 8-arc transitive graphs," Combinatoria 3 (1981), 309-311.

22. S. Zhou, "Almost covers of 2-arc transitive graphs," Combinatorica 24 (2004), 731-745. 